\title{
Effect of antenna-medium coupling in the analysis of ground-penetrating radar data
}

\author{
Sébastien Lambot ${ }^{1 *}$, Frédéric André ${ }^{1}$, Evert Slob ${ }^{3}$ and Harry Vereecken ${ }^{2}$ \\ ${ }^{1}$ Earth and Life Institute, Environmental Sciences, Université catholique de Louvain \\ ${ }^{2}$ Agrosphere (IBG-3), Institute of Bio- and Geosciences, Forschungszentrum Jülich GmbH, 52425 Jülich, Germany \\ ${ }^{3}$ Department of Geotechnology, Delft University of Technology, Stevinweg 1, 2628 CN Delft, The Netherlands
}

Received August 2011, revision accepted February 2012

\begin{abstract}
Physically-based ground-penetrating radar (GPR) data processing is essential for quantitative characterization of soils and materials. A novel near-field GPR antenna model coupled with layered media Green's functions was used to investigate the effect of antenna-medium coupling in the analysis of GPR data. The radar antennas are modelled using an equivalent set of infinitesimal electric dipoles and characteristic, frequency-dependent, global reflection and transmission coefficients. These coefficients determine through plane wave decomposition, wave propagation between the radar reference plane, point sources and field points. We calibrated an actual commercial $400 \mathrm{MHz}$ time-domain antenna, from which synthetic GPR data sets were generated. We observed that, depending on the model configuration, antenna effects may affect the topography of the objective function in full-waveform inverse problems. In addition, antenna-medium coupling has a significant impact on the medium surface reflection, whether in terms of amplitude or propagation time (which usually defines the so-called time zero). We also showed that an effective source cannot be used for simulating near-field radar data as the antenna-medium coupling strongly depends on medium properties. In this respect, numerical experiments demonstrated promising perspectives for simultaneous estimates of medium permittivity and conductivity from antenna-medium coupling.
\end{abstract}

\section{INTRODUCTION}

Near-field ground-penetrating radar (GPR) techniques for nondestructive imaging and characterization of materials have been subject to intensive research for many years (Slob et al. 2010). A major shortcoming in current knowledge is the modelling of the radar signal, which is necessary for quantitative reconstruction using inversion. Existing GPR data processing techniques usually rely on strongly simplifying assumptions and in particular, neglect antenna effects that include frequency-dependent radiation pattern, gain, phase delay, mutual coupling and coupling with the medium of interest. As a result, current full-waveform inversion schemes still suffer from strong limitations with respect to quantitative retrieval due to the unavailability of correct and efficient electromagnetic models.

Antennas can be modelled using numerical approaches, such as the finite-difference time-domain (FDTD) method (Warren and Giannopoulos 2011; Meles et al. 2011), finite element method (FEM) (Ilic et al. 2009), or the method of moments (MoM) (Craeye et al. 2009). Yet, numerical approaches need significant computing resources and suffer from inherent differences between real and conceptualized antenna models. For

* sebastien.lambot@uclouvain.be instance, Warren and Giannopoulos (2011) used a three-dimensional (3D) FDTD approach, through which the different parts of a bowtie antenna were reproduced in a discretized model. Although relatively good modelling results were obtained for data collected over different emulsions, significant modelling errors could still be observed.

More efficient techniques are based on electric field integral equation (EFIE) formulations (Sarkar and Taaghol 1999; Alvarez et al. 2007; Craeye and Gonzalez-Ovejero 2011), through which antennas are emulated using a set of infinitesimal dipoles and field points. The parametrization of these dipoles to properly describe real antenna radiation patterns is however not straightforward (Alvarez et al. 2007; Serhir et al. 2010). In addition, such formulations do not directly account for wave propagation between point sources or field points and the radar transmission line reference plane and hence, antenna-target interactions and mutual coupling are not directly accounted for. For instance, Gentili and Spagnolini (2000) modelled a GPR horn antenna at some distance over a 3D layered medium using an array of frequency-independent source dipoles and a feeding line characteristic impedance. Yet, with this approach the multiple reflections between the antenna terminal section and the target could not be accounted for. 
For the particular case of far-field GPR with applications to planar layered media, Lambot et al. (2004) proposed a closedform, frequency-domain, radar equation that simultaneously accounts for (1) all antenna effects through frequency-dependent global reflection and transmission coefficients and (2) wave propagation in layered media through 3D Green's functions. This integrated model relies on the assumption that the spatial distribution of the backscattered field tends to a plane wave over the antenna aperture. A high level of accuracy was reached for describing radar data and retrieving the medium electrical properties in a series of hydrogeophysical and engineering applications (Lambot et al. 2004; Minet et al. 2010; Soldovieri et al. 2011; Patriarca et al. 2011). In addition, the validity of this model being theoretically independent of frequency, the approach also applies to electromagnetic induction (Moghadas et al. 2010). More recently, this far-field model was generalized to near-field conditions through a plane wave decomposition, thereby providing, to the best of our knowledge, one of the most effective and accurate ways to model radar data for the particular case of wave propagation in layered media (Lambot and André 2012) ('Method and device for characterization of physical properties of a target volume by electromagnetic inspection' by S. Lambot, EU Patent Application N 11160917.8 - 2213).

In this paper, this novel near-field radar model was coupled with layered media Green's functions to investigate the effect of antenna-medium coupling in the analysis of GPR data. A timedomain radar antenna was calibrated in laboratory conditions for providing realistic antenna characteristic coefficients. The antenna model was then used to generate synthetic GPR data for media with different electrical properties. In particular, we analysed the effect of the antenna on the topography of objective functions in full-waveform inverse problems as well as on the first arrival amplitude and propagation time that are usually used in straight ray-based GPR data processing. Finally, we analysed to what extent antenna-medium coupling may affect the wave that is transmitted into a medium through the use of an effective source.

\section{NEAR-FIELD RADAR MODEL}

\section{Antenna equation}

An efficient approach for modelling radar antennas in near-field conditions is to consider an equivalent set of infinitesimal electric dipoles $\left(J_{x, n}, n=1 \cdots N\right)$ for the source and a set of points $\left(E_{x, m}, m=1 \cdots N\right)$ where the field is calculated for the receiver, which is based on the superposition principle (Gentili and Spagnolini 2000; Serhir et al. 2010). As recently proposed by Lambot and André (2012) for the particular case of wave propagation in layered media, wave propagation between point sources or field points and the reference plane of the radar transmission line can be accounted for by means of complex, frequency-dependent global reflection and transmission coefficients. These characteristic coefficients determine the antenna and transmission line internal transmissions and reflections and thereby antenna-medium interactions. The number of points to consider for the point sources and receivers depends on the complexity of the scattered field distribution, which is intrinsically decomposed into a number of plane waves. Although mutual coupling between the transmitting and receiving antennas is not explicitly included in the proposed model, it can be shown that it is implicitly accounted for thanks to the linearity of Maxwell's equations. The antenna model can be formulated in a closed form in the frequency domain as follows (Lambot and André 2012):

$S=\frac{b}{a}=T_{0}+\left|T_{s, 1} T_{s, 2} \cdots T_{s, N}\right|\left(\left(A^{H} A\right)^{-1} A^{H} b\right)$

with

$\mathbf{A}=\left[\begin{array}{cccc}R_{s, 1} G_{11}^{0} & R_{s, 2} G_{12}^{0} & \cdots & R_{s, N} G_{1 N}^{0} \\ R_{s, 1} G_{21}^{0} & R_{s, 2} G_{22}^{0} & \cdots & R_{s, N} G_{2 N}^{0} \\ \vdots & \vdots & & \vdots \\ R_{s, 1} G_{N 1}^{0} & R_{s, 2} G_{N 2}^{0} & \cdots & R_{s, N} G_{N N}^{0}\end{array}\right]-\mathbf{I}_{\mathbf{N}}$

and

$\mathbf{b}=-\left[\begin{array}{cccc}T_{i, 1} G_{11} & T_{i, 2} G_{12} & \cdots & T_{i, N} G_{1 N} \\ T_{i, 1} G_{21} & T_{i, 2} G_{22} & \cdots & T_{i, N} G_{2 N} \\ \vdots & \vdots & & \vdots \\ T_{i, 1} G_{N 1} & T_{i, 2} G_{N 2} & \cdots & T_{i, N} G_{N N}\end{array}\right]\left[\begin{array}{c}1 \\ 1 \\ \vdots \\ 1\end{array}\right]$

where $S(\omega)$ denotes the radar signal expressed here as the ratio between the backscattered field $b(\omega)$ and incident field $a(\omega)$ at the radar transmission line reference plane, $\omega$ being the angular frequency, $T_{0}(\omega)$ is the global transmission or reflection coefficient of the antenna in free space (referred to by subscript 0 ) for non-zero or zero-offset source-receiver, respectively, $T_{s .}(\omega)$ is the global transmission coefficient for fields incident from a field point onto the radar reference plane, $T_{i, .}(\omega)$ is the global transmission coefficient for fields incident from the radar reference plane onto a point source, $R_{s,}(\omega)$ is the global reflection coefficient for the field incident from the layered medium onto a field point, $I_{N}$ is the $N$-order identity matrix and superscript $H$ denotes the Hermitian (conjugate transpose). The quantities $G_{. .}(\omega)$ and are, respectively, the layered medium Green's functions for fields incident from the source points onto the field points and from the field points onto the field points themselves. The Green's functions and the global reflection and transmission coefficients are complex valued and frequency dependent. The number of source and field points to consider depends on the complexity of the backscattered field distribution over the antenna aperture and represents the order of the plane wave decomposition, which can remain limited (e.g., <10) in practice for the particular case of wave propagation in layered media. For far-field conditions, this number can be reduced to 1 and equation (1) reduces to the farfield radar model of Lambot et al. (2004). Finally, it is noted that because Maxwell's equations formulate the field strengths linearly proportional to the source strength, the global reflection and 


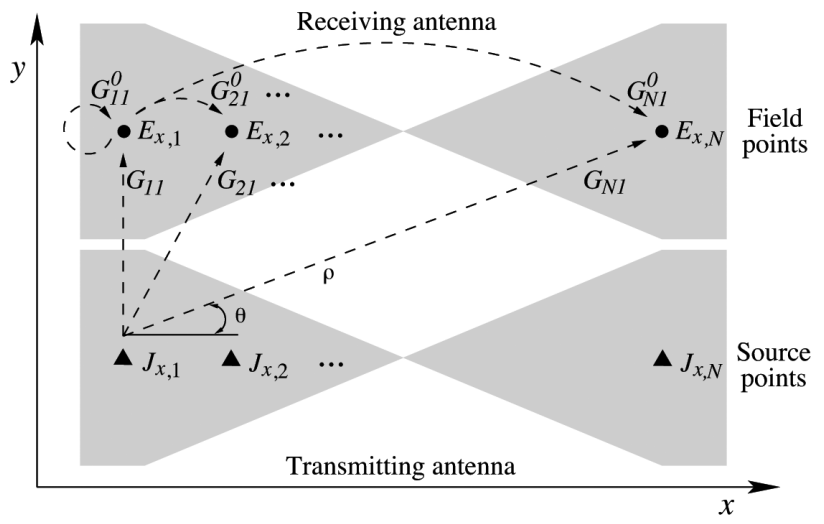

FIGURE 1

Transmitting and receiving antennas modelled using a set of equivalent infinitesimal electric dipole point sources $\left(J_{x,}\right)$ and field points $\left(E_{x_{n}}\right)$ in the $x-y$ plane of a 3D Cartesian system. The dashed lines represent the layered medium Green's functions from the sources to the receivers $(G .$.$) and$ from the receivers, acting as secondary sources, to the receivers $\left(G^{0}\right)$.

transmission coefficients are independent of the medium properties and the total medium response enters equation (1) explicitly as Green's functions between each source and receiver point. We refer to Lambot and André (2012) for additional details on this generalized radar antenna model and its validation.

\section{Layered media Green's functions}

The Green's functions are defined as the scattered $x$-directed electric field $E_{x}$ at the field points for unit-strength $x$-directed electric sources $J_{x}$, an example of which is shown in Fig. 1 for two bowtie antennas. The Green's functions are derived using a recursive scheme to compute the global reflection coefficients of the layered medium in the spectral domain (Knight and Raiche 1982; Slob and Fokkema 2002; Lambot et al. 2004). The transformation back to the spatial domain is performed by evaluating numerically a semi-infinite integral, for which a fast procedure is applied (Lambot et al. 2007). The spatial-domain Green's function is found to be:

$G . .=\frac{1}{8 \pi} \int_{0}^{+\infty} \tilde{G} . .\left(k_{\rho}\right) k_{\rho} d k_{\rho}$

where the spectral Green's function is defined as:

$$
\begin{aligned}
\tilde{G}_{. .}\left(k_{\rho}\right)=[ & J_{0}\left(k_{\rho} \rho\right)\left(\frac{\Gamma_{0} R_{0}^{T M}}{\sigma_{0}+\jmath \omega \varepsilon_{0}}-\frac{\jmath \omega \mu_{0} R_{0}^{T E}}{\Gamma_{0}}\right)- \\
& \left.J_{2}\left(k_{\rho} \rho\right) \cos (2 \theta)\left(\frac{\Gamma_{0} R_{0}^{T M}}{\sigma_{0}+\jmath \omega \varepsilon_{0}}+\frac{\jmath \omega \mu_{0} R_{0}^{T E}}{\Gamma_{0}}\right)\right] \exp \left(-2 \Gamma_{0} h_{0}\right)
\end{aligned}
$$

In this expression, $J_{0}$ and $J_{2}$ are, respectively, the first kind zeroand second-order Bessel's functions, $\rho$ and $\theta$ are, respectively, the distance and angle in the $x y$-plane between the source and field points (see Fig. 1), subscript 0 refers to the upper half-space (freespace), $h_{0}$ is the distance between the source/receiver points and the first medium interface, $R^{T M}$ and $R^{T E}$ are, respectively, the transverse magnetic (TM) and transverse electric (TE) global reflection coefficients accounting for all reflections in the layered medium, $\Gamma$ is the vertical wavenumber defined as $\Gamma=\sqrt{k_{\rho}^{2}-k^{2}}$, whilst $k^{2}=\omega^{2} \mu\left(\varepsilon-\frac{\jmath \sigma}{\omega}\right)$ with magnetic permeability $\mu$, dielectric permittivity $\varepsilon$ and electrical conductivity $\sigma$.

\section{Determination of the antenna characteristic coefficients}

The antenna characteristic coefficients can be determined by solving the non-linear system of equations (1) in which the quantities $S(\omega)$ can be measured and the corresponding Green's functions $G(\omega)$ can be calculated for known medium configurations. In this respect, performing measurements in both near- and farfield conditions over a perfect electrical conductor (PEC) is in particular practical and permits unique estimates of the antenna transmission and reflection functions. The inverse calibration procedure is however not straightforward (see Lambot and André 2012 for the detailed procedure).

In this study, we used a time-domain GPR system (model SIR-20, Geophysical Survey Systems, Inc., GSSI, Salem, Massachusetts, USA) equipped with $400 \mathrm{MHz}$ centre frequency transmitting and receiving bowtie antennas. Radar measurements were performed with the antenna situated at 100 different heights $h_{0}$ above a copper plane $\left(3 \times 3 \mathrm{~m}^{2}\right.$ area), assumed as an infinite PEC. The height of the antenna aperture above the copper plane varied from 0 to about $0.76 \mathrm{~m}$, with an increasing height step varying from $0.001-0.178 \mathrm{~m}$. The time range was $50 \mathrm{~ns}$ and 512 samples per scan were recorded, with 16 bits per sample. For each measurement height, about 1000 traces were collected and averaged. The raw radar data were transformed into the frequency domain using the fast Fourier transform (FFT). Only data between 60-900 MHz, with the highest signal-to-noise ratio, were considered, resulting in 43 observation frequencies (frequency step of $20 \mathrm{MHz}$ ). In addition to the measurements above the copper plane, a measurement was also performed in free space conditions with the antenna held at about $3 \mathrm{~m}$ above the ground and directed towards the sky, thereby providing a direct measurement for $T_{0}(\omega)$. The bowtie antennas were modelled using an equivalent set of 8 point sources and 8 field points, respectively, evenly distributed over the antenna aperture along a single axis. Symmetry of the antenna was assumed, which reduced the number of complex unknowns in the antenna calibration inverse problem.

Figure 2 shows the measured and modelled radar data over the copper sheet in the time domain $(s(t))$. The antenna internal reflections can be clearly observed as well as the copper sheet reflection that appears at larger times for increasing heights $\left(h_{0}\right)$. A first-order multiple between the antenna and the copper sheet can also be observed. The measured and modelled data agree remarkably well, with differences in terms of signal amplitude that are less than 5\%. The amplitude of the observed errors, as depicted in Fig. 2(c), does not depend on the antenna height above the PEC and is similar in both the near- and far-field regions. As the structure of these errors closely follows the PEC 


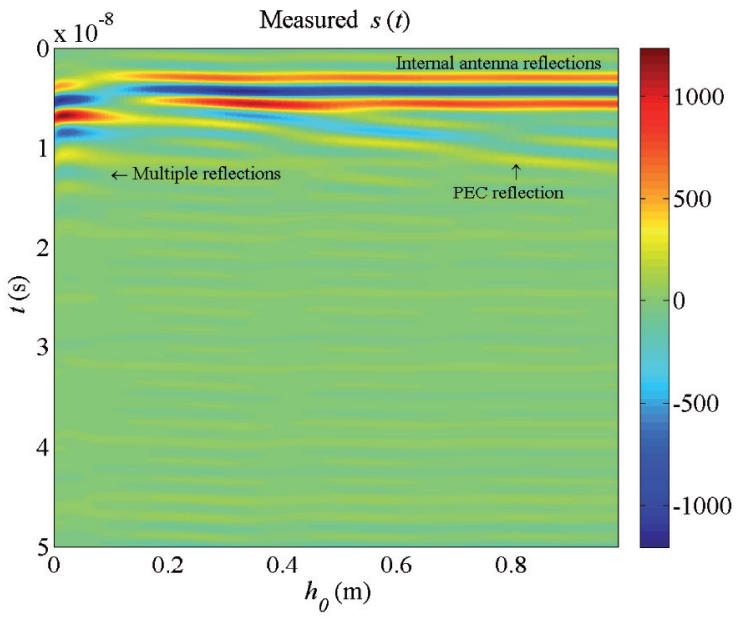

(a)

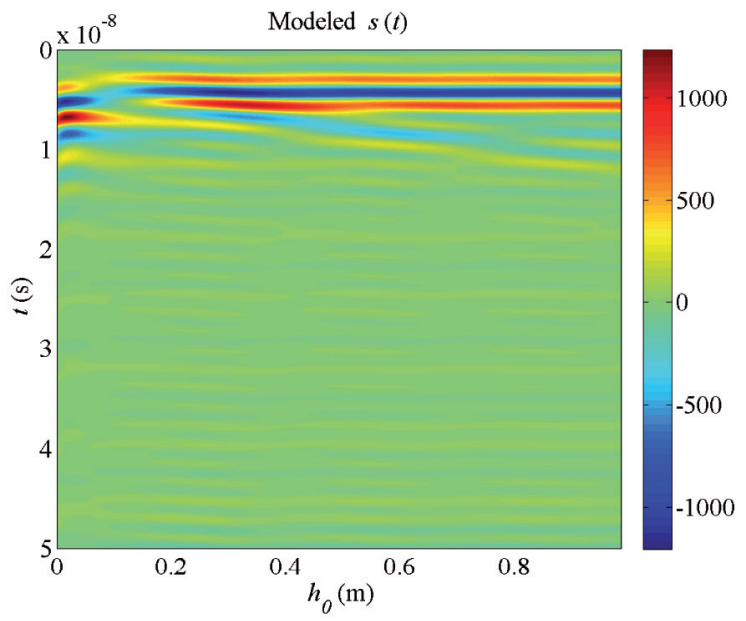

(b)

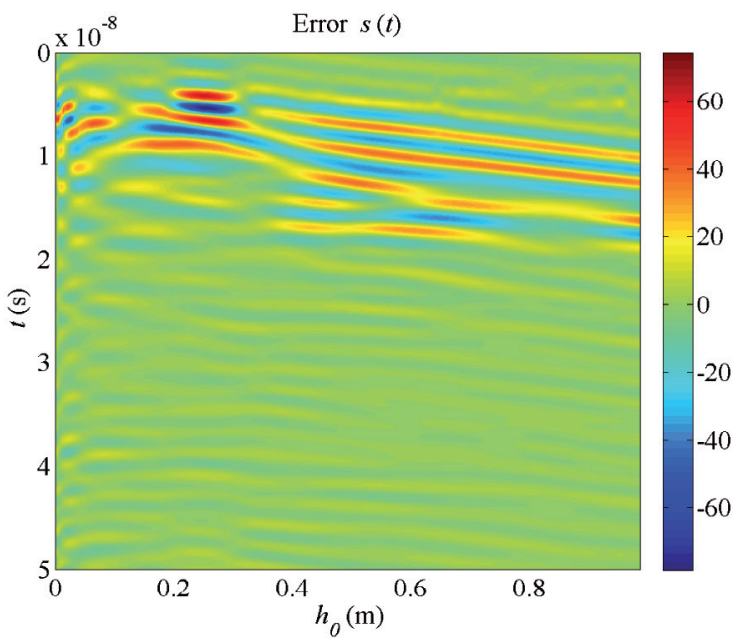

(c)

FIGURE 2

Measured (a) and modelled (b) radar data expressed in the time domain $(s(t))$ for the antenna at different heights $\left(h_{0}\right)$ over the copper sheet. (c) Difference between the measured and modelled data. reflection, it may partly result from a slight time shift between the measured and modelled reflections, which may partly be attributed to instrument drift.

Figure 3 represents the amplitude and phase of the global transmission coefficients $T_{i}$. $(\omega)$ as a function of frequency for the incident field relative to the first four point sources corresponding to one half of the transmitter bowtie shown in Fig. 1. The amplitude shows a relatively smooth, Gaussian-type behaviour with frequency, yet including a drop around $350 \mathrm{MHz}$. This drop is expected to correspond to a relatively strong antenna internal reflection but it may also result from a calibration error for these frequencies. The phase variation with frequency is nearly linear, indicating that the antenna is not significantly dispersive. The physical meaningfulness of the calibration leading to continuous functions with respect to frequency is partly strengthened by the fact that each frequency constitutes an independent calibration problem. Indeed, this continuity with respect to frequency might not have been observed in case the optimization would have led to local solutions. Finally, it is worth recalling that the antenna characteristic global reflection and transmission coefficients are independent of the medium and hence, the calibration over the PEC is valid for application to any layered media.

\section{Numerical experiments}

In order to investigate the effect of the antenna in GPR data analysis, we generated synthetic Green's functions $(G)$ and corresponding radar measurements including the antenna model $(S)$ for wave propagation in a half-space or two-layered medium subject to different permittivities and conductivities that were assumed to be constant with frequency. Hence, the so-called measurements $(S)$ were simulated using the calibration of the actual radar antenna (see above), thereby providing realistic
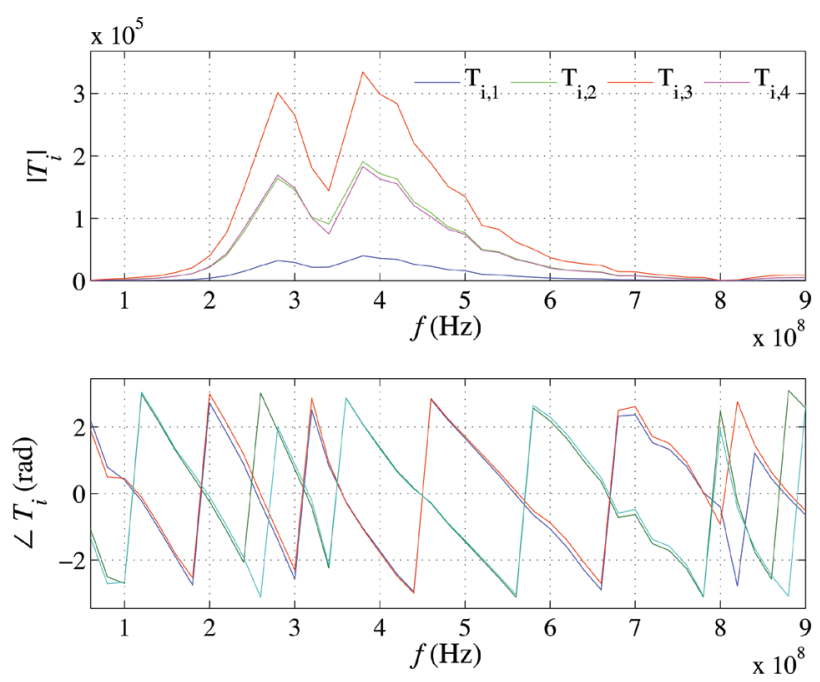

FIGURE 3

Amplitude and phase of the global transmission coefficients $T_{i . .}(\omega)$ as a function of frequency $f$ for the incident field relative to the four first point sources. 

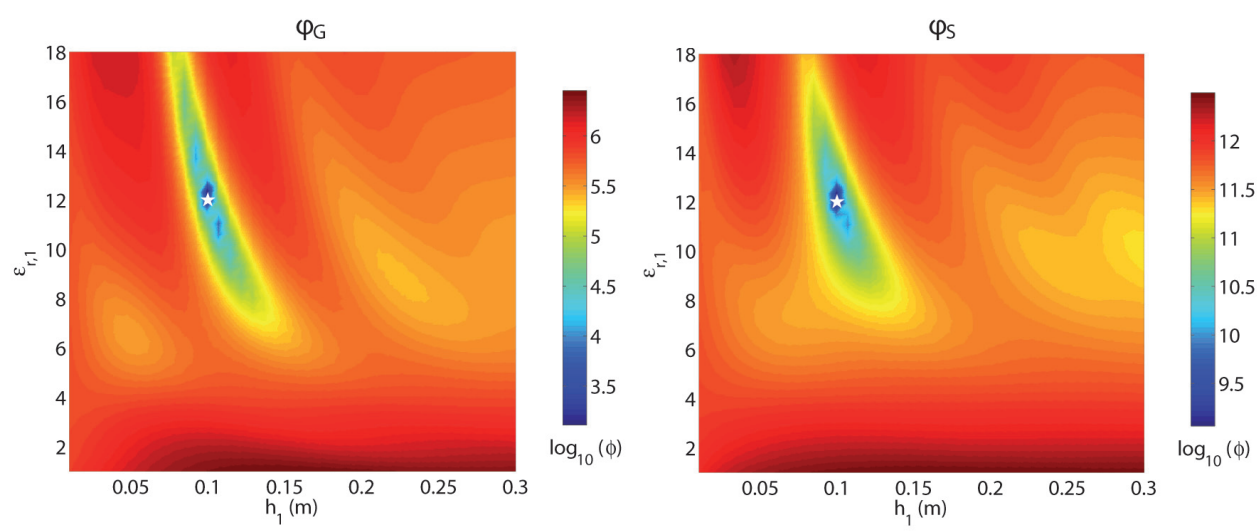

FIGURE 4

Response surfaces of the objective function for synthetic GPR Green's functions $\left(\phi_{G}\right)$ and measurements $(\phi)$ considering $400 \mathrm{MHz}$ antenna centre frequency. The antenna is on a two-layered medium. The white star represents the true values of the parameters. (a)

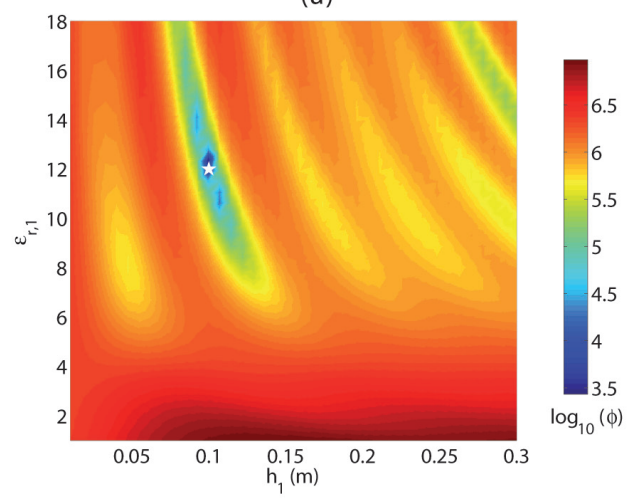

(c)

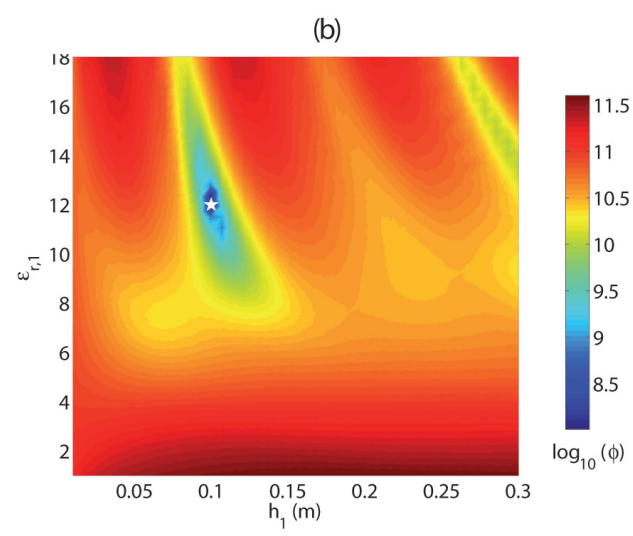

(d)

radar data. The conductivities were related to the permittivities through the Ledieu et al. (1986) and Rhoades et al. (1976) models for realistic values for the soil when subject to a range of water content $(0.05 \cdots 0.31)$. Hence, the medium electrical conductivities were different from zero for all scenarios, thereby non-linearly increasing with permittivity. The relative permittivity varied in the range, $\varepsilon_{\mathrm{r}}=2 \ldots 14$, while the corresponding conductivity varied in the range $\sigma=0.001 \ldots 0.015 \mathrm{~S} / \mathrm{m}$.

\section{Effect of the antenna in full-waveform inverse problems}

For this analysis, the model configuration was two-layered with $\varepsilon_{r, 1}=12, \varepsilon_{r, 2}=9, \sigma_{1}=0.012 \mathrm{~S} / \mathrm{m}, \sigma_{2}=0.006 \mathrm{~S} / \mathrm{m}$, and $h_{1}=0.1 \mathrm{~m}$, where subscripts 1 and 2 denote the first and second layer, respectively. An inverse problem was formulated in terms of least-squares optimization and the corresponding objective functions were plotted. The objective functions were defined as follows, respectively:

$$
\begin{aligned}
& \phi_{s}(b)=\left|S^{*}-S\right|^{T}\left|S^{*}-S\right| \\
& \phi_{G}(b)=\left|G^{*}-G\right|^{T}\left|G^{*}-G\right|
\end{aligned}
$$

where $\mathbf{b}$ is the parameter vector to be estimated, $S^{*}=S^{*}(\omega)$ and $\mathrm{S}=\mathrm{S}(\omega, \mathbf{b})$ are the vectors containing, respectively, the observed (synthetic) and simulated radar data and $G^{*}=G_{11}^{*}(\omega)$ and
$\mathrm{G}=\mathrm{G}_{11}(\omega, \mathbf{b})$ are the vectors containing, respectively, the observed and simulated Green's functions. These vectors are arranged versus frequency. It is worth noting that in practice, the Green's function inversion cannot be performed without an antenna model relating the Green's functions to the raw radar data $\left(\mathrm{S}^{*}\right)$.

Figure 4 shows response surfaces of the objective function for both $S$ - and $G$-based optimization problems (following equations (6 and 7)) considering 43 (Fig. 4a,b) and 5 (Fig. 4c,d) frequencies in the range 60-900 MHz. First, we observe a negative correlation (banana-shaped valley) between the permittivity $\varepsilon_{\mathrm{r}, 1}$ and thickness $h_{1}$ of the top layer. This correlation is due to the fact that an infinite number of combinations $\varepsilon_{\mathrm{r}, 1}-h_{1}$ can lead to the same propagation time between the two interfaces surrounding the top layer 1 . The solution remains however unique thanks to the amplitude information, a thicker layer leading to more attenuation due to spherical divergence and electrical losses ( $\sigma=0.01 \mathrm{~S} / \mathrm{m}$ in this example). The objective function topography is similar for both $G$ and $S$ when considering the 43 frequencies, with a slightly larger valley for $G$ denoting a larger uncertainty in parameter retrieval. For the 5-frequency case, the inverse problem is less well-posed and a second valley appears due to the decreased information content in the radar data. In addition, the $G$ response surface shows a much larger number of local minimum valleys and hence, increased uncertainty in the 


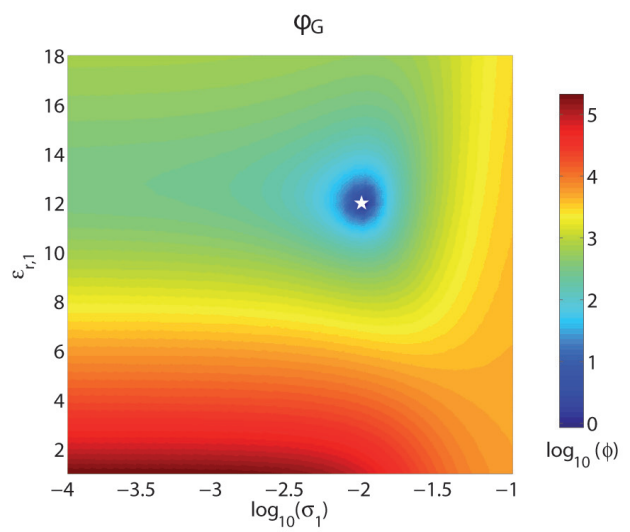

(a)

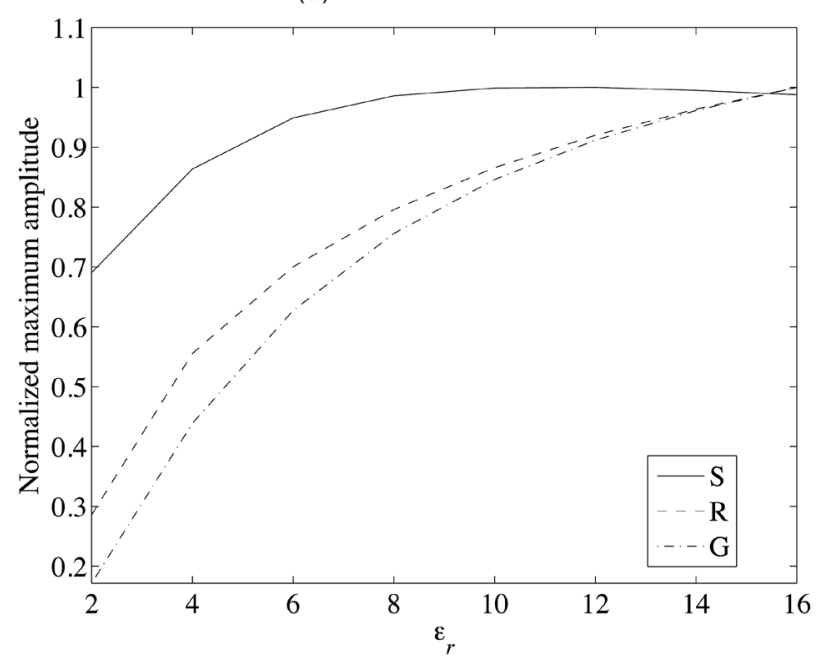

FIGURE 6

Normalized maximum amplitude (peak-to-peak) of a half-space medium surface reflection as a function of the medium permittivity. The amplitude was derived from the Fresnel reflection coefficient $(R)$, the Green's function $(G)$ and the full-model including the antenna effects $(S)$, respectively.

parameters and a more complex optimization problem. In these particular examples, the antenna-medium coupling therefore permitted to increase the information content in the radar data through an increased sensitivity of the radar signal to the toplayer medium properties, as shown by the objective function topography, i.e., the global minimum is better defined as a result of the antenna.

Figure 5 shows another example of objective function topography assuming in this case a single layer (half-space medium) and a centre frequency of $40 \mathrm{MHz}\left(\varepsilon_{r, 1}=12\right.$ and $\left.\sigma_{1}=0.01 \mathrm{~S} / \mathrm{m}\right)$. As transfer functions for this case were not available, we scaled the $400 \mathrm{MHz}$ antenna characteristic coefficients in frequency (virtual antenna). First, we observe a well-defined minimum in the $\varepsilon_{r, 1}-\sigma_{1}$ parameter plane, for both $G$ and $S$. This highlights the potential for the application of the proposed radar model as this means it can theoretically be used for the simultaneous
$\varphi_{\mathrm{S}}$

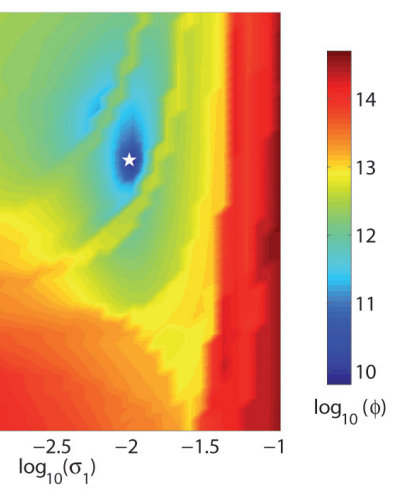

(b)
FIGURE 5

Response surfaces of the objective function for synthetic GPR Green's functions $\left(\Phi_{G}\right)$ and measurements $\left(\Phi_{S}\right)$ considering $40 \mathrm{MHz}$ antenna centre frequency. The antenna is on a half-space medium. The white star represents the true values of the parameters.

retrieval of both quantities for this half-space configuration, thereby bridging the gap between GPR and electromagnetic induction (EMI) sensitivities in this frequency range. In this respect, the parameters $\varepsilon_{r, 1}$ and $\sigma_{1}$ are not significantly correlated. Yet, for the $S$ case, the topography of the objective function contains local minima around the global minimum, which are not present when not accounting for the antenna effects ( $G$ case). Although the antenna dealt with in this example is virtual, this shows the importance of analysing an inverse problem with a correct GPR model. It is in particular relevant to the choice of the optimization strategy (e.g., local versus global), quantification of uncertainty and information content or model sensitivity to the medium parameters.

\section{The surface reflection amplitude}

Simplified analyses of GPR waveforms typically based on straight-ray propagation include the estimation of the medium surface permittivity from early-time arrivals or the surface reflection amplitude and corresponding Fresnel reflection coefficient (R) (Chanzy et al. 1996; Pettinelli et al. 2007). Indeed, the larger the permittivity of a medium, the larger the reflection amplitude is expected to be. This relationship is depicted in Fig. 6 where the reflection amplitude for a half-space medium is shown as a function of permittivity $\left(\varepsilon_{r, 1}=2 \cdots 14, \sigma_{1}=0.001 \cdots 0.015 \mathrm{~S} / \mathrm{m}\right)$. The frequency range was $60-900 \mathrm{MHz}$ with a frequency step of $20 \mathrm{MHz}$ (corresponding to the actual antenna calibration). Although such approaches may lead to relatively good results in far-field conditions (Lambot et al. 2006), significant errors may be introduced when operating in the near-field and in particular with the antenna in contact with the medium. When derived from Green's functions ( $G$ in Fig. 6), the behaviour of the reflection amplitude with respect to permittivity slightly deviates from the Fresnel reflection coefficient derived amplitude due to spherical divergence in wave propagation and electrical conductivity effects, i.e., the reflection coefficient is not a Dirac delta function of time as would be the case for 1D lossless propagation. Neglecting these effects may lead to errors larger than 1 in terms of absolute relative permittivity estimation, which corresponds, for instance, roughly to $2 \%$ of error in terms of absolute soil 
water content. When including the antenna effects and in particular antenna-medium coupling (see $S$ in Fig. 6), the errors become significantly much larger, depending on the contrast between the antenna impedance with respect to the medium properties and antenna distortion effects on the pulse shape. In addition, in this example, we observe that the sensitivity of the surface reflection amplitude with respect to the permittivity is significantly decreased, as a result of the antenna interferences.

\section{The surface reflection time}

In common GPR approaches, it is usually assumed that the time at which the surface reflection occurs exactly corresponds to the reflection interface. This time, or the so called time-zero defined from this surface reflection (Yelf 2004), is then used to calculate the propagation time to a deeper interface and derive the corresponding medium permittivity (or layer thickness when the permittivity is known). Actually, due to the interferences caused by the antenna internal reflections and antenna-medium coupling, the reflections observed in the time domain are distorted and do not exactly correspond to the reflections in the space domain. In addition, for 3D wave propagation, the reflection coefficient is not a Dirac function of time (i.e., the reflection of a Dirac pulse does not lead to a Dirac reflection when representing the radar data in the time domain).

Figure 7(a) shows simulated radar data $(S)$ as a function of different permittivities and corresponding conductivities (see above) for a half-space medium. We clearly observe the nonnegligible effect of permittivity/conductivity on the reflection

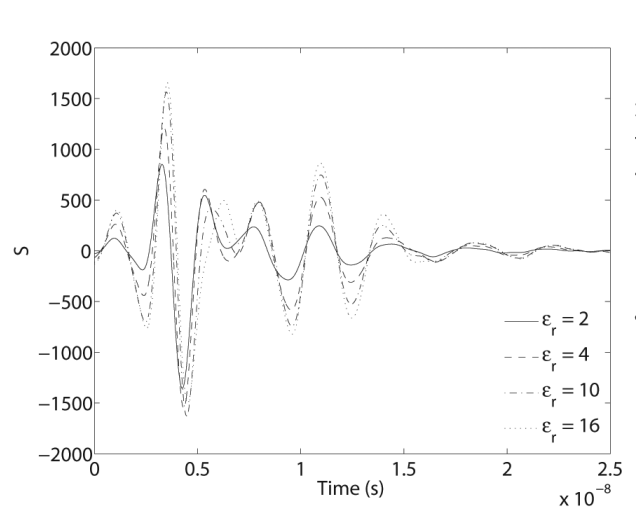

(a)

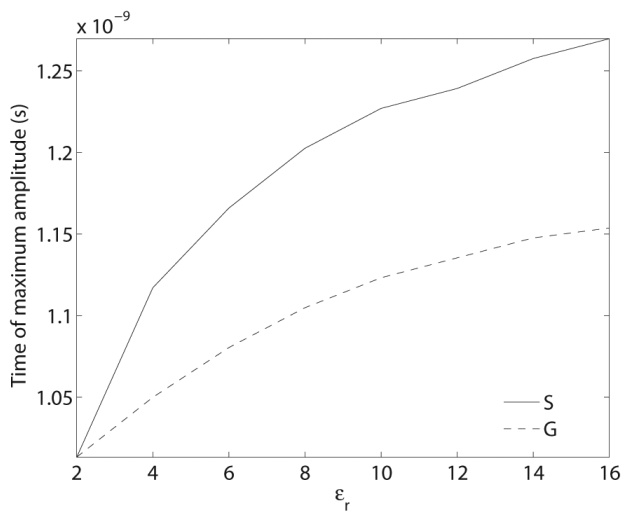

(b)
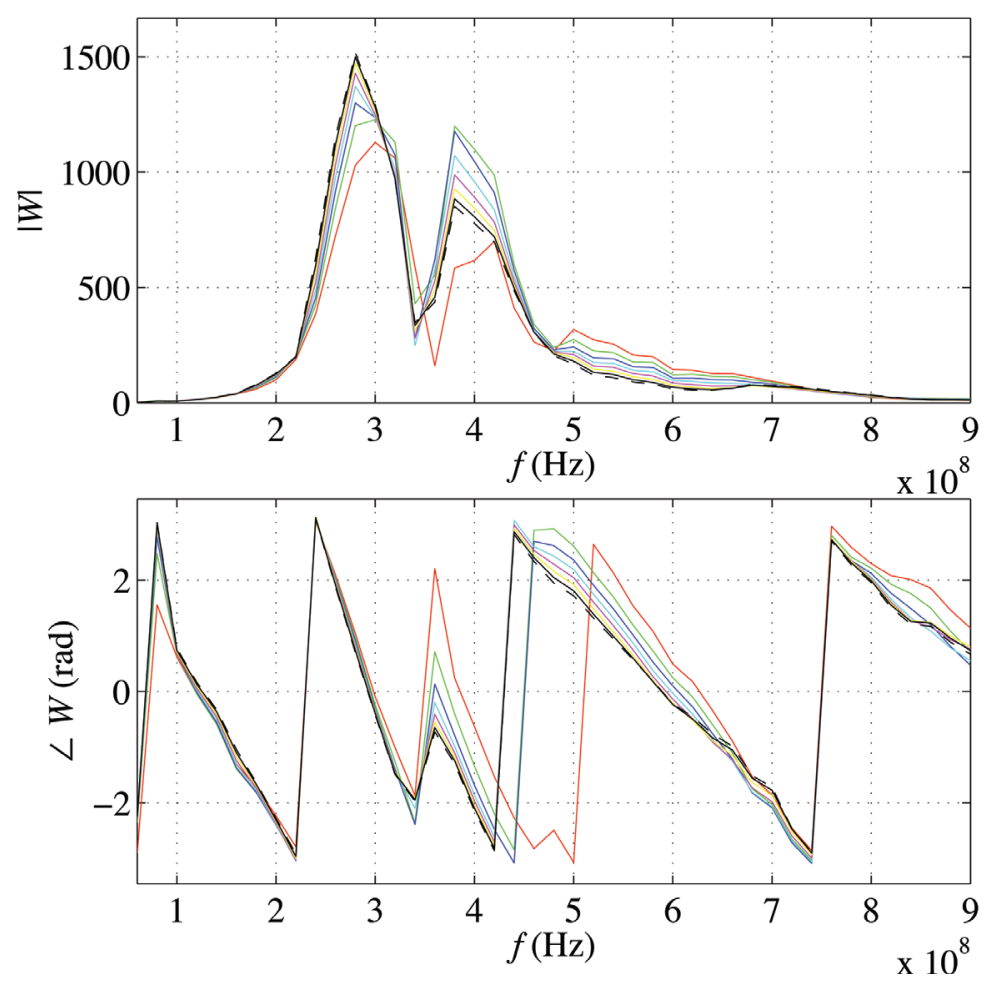

FIGURE 7

(a) Simulated radar data as a function of different permittivities and corresponding conductivities. (b) Time of the first interface reflection as a function of the medium permittivity for a halfspace model configuration.

\section{FIGURE 8}

Effective GPR source in the frequency domain as a function of the medium permittivity. 
time, whether in terms of maximum amplitude or beginning reflection detection. Figure 7(b) shows the time at which the first interface reflection occurs, picked up from the maximum amplitude, as a function of the permittivity for both $S$ and $G$. For the Green's function model $G$, the reflection time is not constant and presents a variation range of about $0.15 \mathrm{~ns}$ (due to spherical divergence and electrical conductivity effects). When accounting for the antenna $(S)$, the variation range reaches about $0.30 \mathrm{~ns}$. Assuming a second interface at $50 \mathrm{~cm}$ depth, for instance, with an actual medium relative permittivity of 6 , this would result in an error of about 0.5 in terms of absolute permittivity estimation. The error may be larger or smaller, depending on the distances, medium properties and particular antenna.

\section{The effective source model}

It is well-known that the GPR wave that is actually transmitted into the ground depends on the medium properties as a result of the antenna-medium coupling. Yet, an effective source $W$ may be defined in the frequency domain as the ratio between the Green's function and the corresponding radar signal as empirical simplification. Figure 8 shows the amplitude of such an effective GPR source $W$ in the frequency domain as a function of the medium permittivity. It can be observed that although the general behaviour of the effective source is similar to the antenna transmission coefficient functions depicted in Fig. 3, the amplitude may change up to $70 \%$, depending on frequency. Significant phase shifts can also be observed. These results in particular highlight the need for physically-based antenna models in GPR data processing for quantitative characterization of material properties.

\section{CONCLUSIONS AND PERSPECTIVES}

We used a novel near-field radar antenna model coupled with layered media Green's functions to investigate the importance of antenna-medium coupling in the analysis of GPR data. The analysis was based on the calibration of an actual commercial $400 \mathrm{MHz}$ time-domain antenna, for which a successful comparison between simulated and measured radar data was obtained. First, we observed that, depending on the layered model configuration and information content in the radar data with respect to the parameters of interest, antenna effects may in some cases significantly change the topography of the objective functions dealt with. It is therefore important to account for the antenna when formulating GPR inverse problems, although such effects are not always significant. Second, we showed that antennamedium coupling has a significant impact on the medium surface reflection, whether in terms of amplitude or propagation time. In this respect, the well-known time-zero that is usually defined from the surface reflection is actually variable, depending on the medium properties. It is worth noting that with the proposed radar model, the time-zero is fixed and corresponds to the radar reference plane. Finally, we illustrated the significant effect of medium properties on an effective source, which defines the actual wave that is transmitted into the medium.
In addition to highlighting the importance of accounted for radar antennas in various GPR data processing approaches, we also introduced the promising perspective of applying the proposed radar model to relatively low-frequency antennas (e.g., $40 \mathrm{MHz}$ ) for the simultaneous estimation of the medium permittivity and conductivity, through full-wave inversion focused on the antenna-medium coupling reflection.

\section{ACKNOWLEDGEMENTS}

This research was supported by the Fonds de la Recherche Scientifique (FNRS, Belgium) and the DIGISOIL project financed by the European Commission under the 7th Framework Programme for Research and Technological Development, Area 'Environment', Activity 6.3 'Environmental Technologies'.

\section{REFERENCES}

Alvarez Y., Las-Heras F. and Pino M.R. 2007. Reconstruction of equivalent currents distribution over arbitrary three-dimensional surfaces based on integral equation algorithms. IEEE Transactions on Antennas and Propagation 55(12), 3460-3468.

Chanzy A., Tarussov A., Judge A. and Bonn F. 1996. Soil water content determination using digital ground penetrating radar. Soil Science Society of America Journal 60, 1318-1326.

Craeye C., Gilles T. and Dardenne X. 2009. Efficient full-wave characterization of arrays of antennas embedded in finite dielectric volumes. Radio Science 44.

Craeye C. and Gonzalez-Ovejero D. 2011. A review on array mutual coupling analysis. Radio Science 46, 25.

Gentili G.G. and Spagnolini U. 2000. Electromagnetic inversion in monostatic ground penetrating radar: TEM horn calibration and application. IEEE Transactions on Geoscience and Remote Sensing 38(4), 1936-1946.

Ilic M.M., Djordjevic M., Ilic A.Z. and Notaros B.M. 2009. Higher Order Hybrid FEM-MoM Technique for Analysis of Antennas and Scatterers. IEEE Transactions on Antennas and Propagation 57(5), 1452-1460.

Knight J.H. and Raiche A.P. 1982. Transient electromagnetic calculations using the Gaver-Stehfest inverse Laplace transform method. Geophysics 47, 47-50.

Lambot S. and André F. 2012. Full-wave modeling of near-field radar data to reconstruct planar layered media. IEEE Transactions on Geoscience and Remote Sensing. Submitted.

Lambot S., Slob E.C., van den Bosch I., Stockbroeckx B. and Vanclooster M. 2004. Modeling of ground-penetrating radar for accurate characterization of subsurface electric properties. IEEE Transactions on Geoscience and Remote Sensing 42(11), 2555-2568.

Lambot S., Slob E. and Vereecken H. 2007. Fast evaluation of zero-offset Green's function for layered media with application to ground-penetrating radar. Geophysical Research Letters 34, L21405, doi:21410.21029/22007GL031459.

Lambot S., Weihermüller L., Huisman J.A., Vereecken H., Vanclooster M. and Slob E.C. 2006. Analysis of air-launched ground-penetrating radar techniques to measure the soil surface water content. Water Resources Research 42, W11403, doi:11410.11029/12006WR005097.

Ledieu J., De Ridder P., De Clercq P. and Dautrebande S. 1986. A method of measuring soil moisture by time domain reflectometry. Journal of Hydrology 88, 319-328.

Meles G., Greenhalgh S., van der Kruk J., Green A. and Maurer H. 2011. Taming the non-linearity problem in GPR full-waveform inversion for high contrast media. Journal of Applied Geophysics 73(2), 174-186. 
Minet J., Lambot S., Slob E.C. and Vanclooster M. 2010. Soil Surface Water Content Estimation by Full-Waveform GPR Signal Inversion in the Presence of Thin Layers. IEEE Transactions on Geoscience and Remote Sensing 48(3), 1138-1150.

Moghadas D., André F., Vereecken H. and Lambot S. 2010. Efficient loop antenna modeling for zero-offset, off-ground electromagnetic induction in multilayered media. Geophysics 75(4), WA125-WA134.

Patriarca C., Lambot S., Mahmoudzadeh M.R., Minet J. and Slob E. 2011. Reconstruction of sub-wavelength fractures and physical properties of masonry media using full-waveform inversion of proximal penetrating radar. Journal of Applied Geophysics 74(1), 26-37.

Pettinelli E., Vannaroni G., Di Pasquo B., Mattei E., Di Matteo A., De Santis A. and Annan P.A. 2007. Correlation between near-surface electromagnetic soil parameters and early-time GPR signals: An experimental study. Geophysics 72(2), A25-A28.

Rhoades J.D., Raats P.A.C. and Prather R.J. 1976. Effects of liquid-phase electrical conductivity, water content, and surface conductivity on bulk soil electrical conductivity. Soil Science Society of America Journal 40, 651-655.

Sarkar T.K. and Taaghol A. 1999. Near-field to near/far-field transformation for arbitrary near-field geometry utilizing an equivalent electric current and MoM. IEEE Transactions on Antennas and Propagation 47(3), 566-573.

Serhir M., Besnier P. and Drissi M. 2010. Antenna Modeling Based on a Multiple Spherical Wave Expansion Method: Application to an Antenna Array. IEEE Transactions on Antennas and Propagation 58(1), 51-58.

Slob E.C. and Fokkema J. 2002. Coupling effects of two electric dipoles on an interface. Radio Science 37(5), 1073, doi:1010.1029/2001RS2529.

Slob E., Sato M. and Olhoeft G. 2010. Surface and borehole groundpenetrating-radar developments. Geophysics 75(5), A103-A120.

Soldovieri F., Lopera O. and Lambot S. 2011. Combination of Advanced Inversion Techniques for an Accurate Target Localization via GPR for Demining Applications. IEEE Transactions on Geoscience and Remote Sensing 49(1), 451-461.

Warren C. and Giannopoulos A. 2011. Creating finite-difference timedomain models of commercial ground-penetrating radar antennas using Taguchi's optimization method. Geophysics 76(2), G37-G47.

Yelf R. 2004. Where is true time-zero? In: E.C. Slob, J. Rhebergen, and A. Yarovoy (ed). Proceedings of the Tenth International Conference on Ground Penetrating Radar, Delft University of Technology, Delft, The Netherlands. 279-282. 


\section{The Environmental and}

\section{Engineering Geophysical Society}

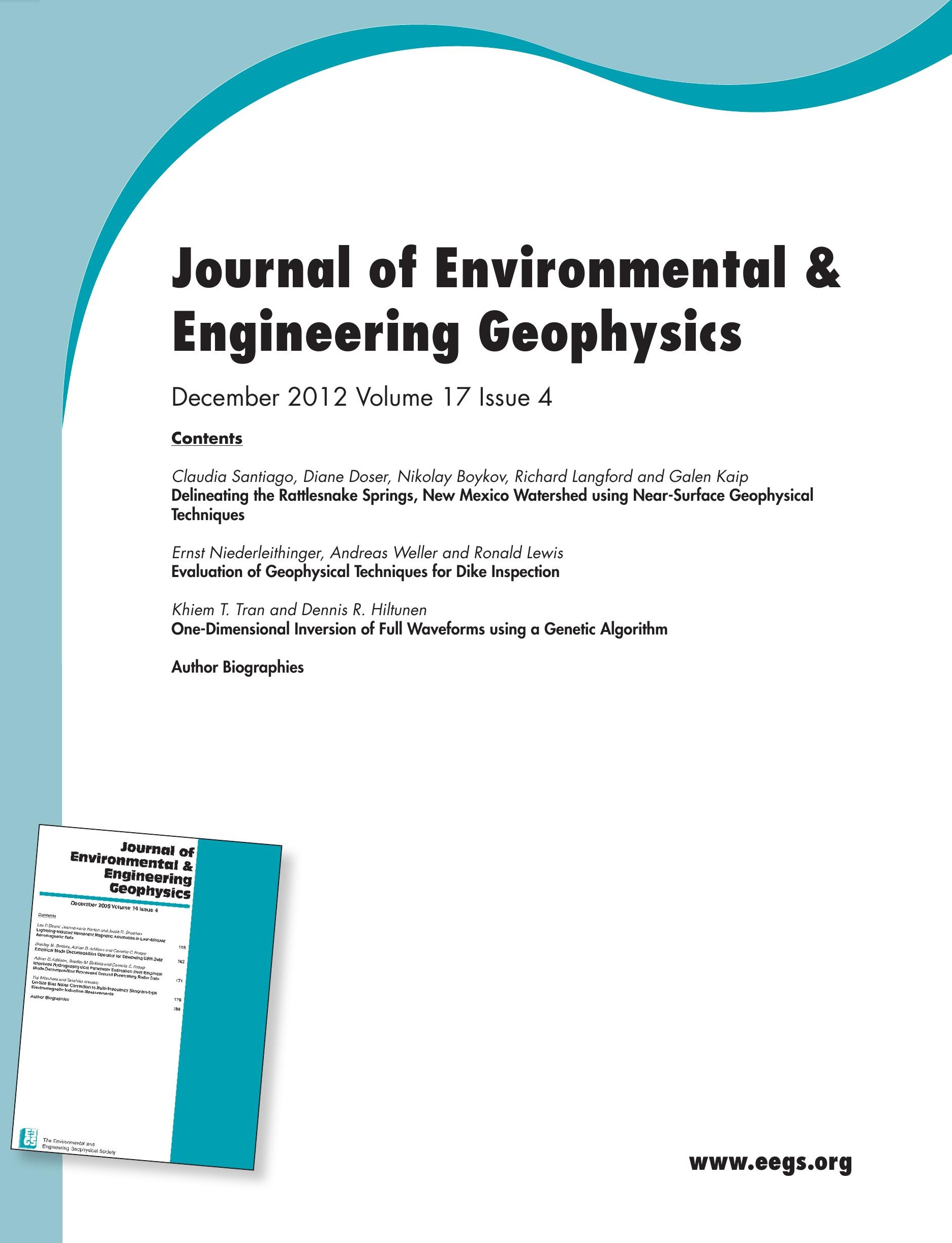

